\title{
Avaliação de primers universais bacteriano em PCR in silico
}

\author{
Evaluation of bacterial universal primers in PCR in silico
}

Luiz Alfredo Torres Sales ${ }^{1}$, João Victor Nogueira Nojosa ${ }^{1}$, Roberval Nascimento Moraes Neto ${ }^{1}$, Alexsander Rodrigues Carvalho Junior ${ }^{1}$, Ruth Flávia Barros Setúbal ${ }^{1}$, Lucas Weba Soares ${ }^{1}$, Luís Claudio Nascimento da Silva², Matheus Silva Alves².

Resumo: As técnicas de biologia molecular são indispensáveis em diversas áreas de pesquisa, dentre elas está a identificação de espécies bacterianas como Staphylococcus aureus, Streptococcus pneumoniae, Pseudomona auruginosa, Klebsiella pneumoniae, Corynebacterium diphtheriae, Mycobacterium tuberculosis e Escherichia coli e para isso utilizam primers universais. Este trabalho busca avaliar a qualidade deste primers universais em amplificar e diferenciar as bactérias descritas. Para isso foi utilizado uma PCR in silico e observados os resultados das amplificações. As amplificações demonstraram uma redundância ou mesmo ineficiência de vários dos primers testados.

Palavras-chave: Primers; Bacterias; PCR in silico.

\begin{abstract}
Molecular biology techniques are indispensable in several researches. Among them the identification of bacterial species such as Staphylococcus aureus, Streptococcus pneumoniae, Pseudomonas auruginosa, Klebsiella pneumoniae, Corynebacterium diphtheriae, Mycobacterium tuberculosis and Escherichia coli, using universal primers. This paper aims to evaluate the quality of this universal primers in amplifying and differentiating the described bacteria. For this purpose, an in silico PCR was used and the results of the amplifications were observed. The amplifications demonstrated a redundancy or even inefficiency of several of the primers tested.
\end{abstract}

Keywords: Primers; Bacteria; PCR in silico.

\footnotetext{
${ }^{1}$ Discente - Universidade Ceuma

${ }^{2}$ Docente - Universidade Ceuma
} 


\section{Introdução}

As principais bactérias de importância clínica e epidemiológica são as espécies Staphylococcus aureus ${ }^{1,2}$, Streptococcus pneumoniae ${ }^{3,4}, \quad$ Pseudomona aeruginosa ${ }^{5}$, Klebsiella pneumoniae 6, Corynebacterium diphtheriae 7, Mycobacterium tuberculosis 8 e Escherichia coli ${ }^{9}$, sendo associadas a diferentes patologias.

O Staphylococcus aureus são cocos gram-positivos com formato de cacho de uva, são encontrados nas mucosas do ser humano. Caso o ambiente na qual se aloja esteja lesionado, a bactéria causará danos nas ligações intercelulares, desmossomos e junções de aderência. Desse modo, ela desenvolve patologias como: foliculite, impetigo, furúnculos, endocardite e bacteremia ${ }^{1,2,10}$. Ademais, o Streptococcus pneumoniae também grampositivos, possuem seus cocos alinhados. Localizam-se na nasofaringe e orofaringe nos seres humanos. Suas cepas causam infecções como: pneumonia, osteomielite e meningite $3,4,11$.

As Pseudomonas aeruginosa são bactérias gram-negativas que apresentam o formato de bacilos. Tem como fatores de virulência as enzimas, adesinas e toxinas (endotoxina A). Desse modo, possibilitam doenças que atingem a pele, o trato respiratório e urinário ${ }^{5}$. A Klebsiella pneumoniae se caracteriza na forma de bacilos gram-negativos. Seu modo de colonização na forma patológica acomete 0 ser humano imunocomprometido e causa quadros de infecção severa nas vias respiratórias ${ }^{6}$.

A espécie bacteriana Corynebacterium difiterae, é um bacilo Gram-positivo, o qual possui como principal fator de virulência e patogenia a exotoxina diftérica. As cepas toxigênicas, ao secretar esta toxina na região nasofaríngea ou cutânea, geram a difteria, uma doença infectocontagiosa aguda ${ }^{7,12,13}$. Já a Mycobacterium tuberculosis é conhecida como bacilo de Koch, espécie bacteriana altamente patogênica e agente causadora da tuberculose. Doença infectocontagiosa que afeta majoritariamente os alvéolos pulmonares, podendo também acometer outros órgãos como os ossos, rins e meninges ${ }^{8,14,15}$. A Escherichia coli por sua vez é uma bactéria, bacilar gram-negativa, natural da microbiota intestinal de muitos mamíferos, porem algumas cepas são patogênicas e responsáveis por infecções na região gastrointestinal, como também infecções urinárias e na meninge ${ }^{16,17}$.

Nesse sentido, a utilização de técnicas de biologia molecular, principalmente a técnica de Reação em Cadeia da Polimerase (PCR), sendo uma ferramenta eficiente na identificação de organismos, tanto no diagnóstico de patologias quanto na pesquisa cientifica. Para que isso aconteça, é necessário a utilização de iniciadores (Primers) que podem identificar vários táxons, apenas sendo necessário a escolha de uma região conservada para o estudo. Em procariotos, geralmente, são desenhados iniciadores na região ribossomal 16S, pois apresenta vários loci conservado para diversas bactérias.

A partir disso, estudos encontrados na literatura apresentam inúmeros primers dito universal para identificação de bactérias. Mas em muitos casos não há amplificação de fragmentos 
contestando assim, a literatura. Com isso, sua utilização tem sido aplicada em estudos, para identificar bactérias de forma geral.

Baseado na importância clínica, científica e na necessidade da realização de técnicas moleculares para um melhor diagnóstico das infecções bacterianas citadas, torna-se necessária uma avaliação da qualidade dos primers universal, visto que são parte fundamental no diagnóstico e no controle de qualidade dos estudos científicos, tanto nas diferentes formas de PCR quanto nos Sequenciamentos de Genoma $^{18,20}$. Este trabalho realizou uma análise in silico de diferentes primers universal descritos por diversos autores, para verificar a sua confiabilidade e sensibilidade.

\section{Metodologia}

O presente trabalho tem característica de pesquisa descritiva, direcionada para análise da eficiência de iniciadores moleculares de replicação de DNA. Inicialmente foram selecionados artigos que apresentavam primers considerados universal para a identificação de bactérias. Esses artigos foram retirados do banco de dados PUBMED - MedLine, e os primers selecionados, descritos na Tabela 1, foram submetidos a uma plataforma

http://insilico.ehu.es/PCR/21 para realizar a PCR in silico, e posterior, verificação de formação de fragmentos para as seguintes bactérias: Klebsiella pneumoniae, Escherichia coli, Corynebacterium diphtheriae, Pseudomonas aeruginosa, Staphylococcus aureus, Streptococcus pneumoniae e Mycobacterium tuberculosis.
Cada par de Primer foi analisado para as sete bactérias descritas anteriormente, e foi verificado se houve a formação de fragmento e o tamanho do fragmento formado.

\section{Resultados}

Os resultados das amplificações dos fragmentos de PCR das bactérias estudadas estão distribuídos na Tabela 1 e figura 1.

\section{Discussões}

A partir dos resultados acima é possível observar uma falha na universalidade dos primers ditos como universais. Dos oito conjuntos de primers estudados, apenas quatro $(50 \%)$ foram eficazes na amplificação de todas as bactérias estudadas (primers: I, IV, V e VIII) outros dois (25\%) - primers II e VII - não conseguiram amplificar nenhuma das oito bactérias estudas na análise in silico.

Os primers do grupo I foram utilizados anteriormente por Ritchie et al 21 com a finalidade de amplificar RNA ribossômicos 16s (rRNA) de diferentes espécies de Lactobacillus e Bifidobacterium com a finalidade de avaliar e caracterizar a microbiota fecal de felinos. Em seu trabalho, eles traçaram um perfil da microbiota dos animais de acordo com os padrões de bandas formados por cada espécie. Esses resultados podem mostrar-se parcialmente conflitosos, visto que o tamanho de pares de bases entre as espécies é bastante próxima, devido ao fato de o rRNA ser altamente conservado nas espécies $^{22}$. 
Além disso, o tamanho de pares de base formadas para a bactéria Lactobacillus é igual às bactérias $E$. coli avaliadas in silico nesse trabalho para 0 mesmo primer. Devido ao fato de E. coli ser um contaminante frequente nas fezes $^{9}$, é possível que essas espécies de Lactobacilus tenham sido confundidas com E. coli, uma vez que a análise foi baseada no fragmento de amplificação.

Já os primers do grupo II e VII, que foram utilizados anteriormente por Saggu e Mishra ${ }^{22}$ e Mao et al23 respectivamente, não amplificaram para nenhuma das sete bactérias testadas in silico neste estudo. Este primer não se mostrou hábil para análises de bactérias patogênicas à espécie humana, uma vez que, pelo menos oito das principais bactérias de importância médica e epidemiológica (citar referencias que sobrarem na introdução) não são identificáveis pelo mesmo.

Os primers utilizados anteriormente por Ito e Suzaki ${ }^{24}$ (III) amplificaram para quatro das sete bactérias testadas $(S$. aureus, $S$. pyogenes, $C$. diphtheriae e $M$. tuberculosis) correspondendo a $57 \%$ de todas as bactérias avaliadas. No entanto, sua cobertura não foi suficiente para as bactérias $P$. auruginosa, $K$. pneumoniae e E. coli, o que torna preocupante sua utilização por exemplo em uma análise ambiental ou de contaminação fecal pela não identificação de $E$. coli ${ }^{9}$. Além disso, é inábil para identificação de possíveis superbactérias em um estudo epidemiológico, como por exemplo a $K$. pneumoniae carbapenemase (KPC) ${ }^{29}$.

Já os primers do grupo IV embora tenham tamanhos de bases parecidos e muito variáveis dentro de uma mesma espécie, mostraram-se eficientes contra todas as sete bactérias analisadas em PCR in silico. O que corrobora com os resultados encontrados por $^{25}$, no qual foi feita a avaliação da qualidade de primers em PCR real time para diferentes bactérias. Entre as bactérias avaliadas, encontram-se diferentes cepas de $P$. aeruginosas, $P$. fluorescens, $P$. putida, $P$. stutzeki, Legionella pneumophila, E. coli, Serratia marcecens, Sthaphycoccus aureus, Staphylococcus epidermidis, Streptococcus pyogenes, Streptococcus mutans, entre outras.

Os primers do grupo $\mathrm{V}$ foram recentemente utilizados para análise de microbiota de camundongos sujeitos a diferentes dietas, visando observar alguma diferença significativa entre camundongos com maior pressão sanguínea sistólica em relação aos normal ${ }^{26}$. Estudos utilizando os mesmos primers em análise da microbiota humana também demonstraram-se eficazes ${ }^{30}$. Logo, podemos concluir que os primers $\mathrm{V}$ foram efetivos na amplificação das cepas consideradas universais in vivo, colaborando com nossos resultados realizados in silico.

Enquanto 0 primer VIII também demonstrou-se efetivo na detecção de bactérias de cunho patológico em nossas análises in silico assim como em análises in vivo realizadas em outros experimentos $^{28}$. Walter e colaboradores, ao utilizarem 0 primer VIII visando a detecção de cepas de Lactobacilus, uma cepa comum na microbiota do trato gastrointestinal humano, obteram resultados positivos. Em seu experimento, Walter produziu grupos de camundongos com e sem Lactobacilus em sua microbiota gastrointestinal. Ao realizar PCR do 
material extraído do estômago destes camundongos utilizando 0 primer VIII, Walter et al foi capaz de detectar diferenças significativas nos padrões de bandas entre os dois grupos testados. Este resultado demonstra a incerteza do primer VIII para uso em diagnóstico de bactérias patologicas, tendo em vista que o mesmo também é capaz de detectar bactérias probióticas.

Por fim, os primers VI apresentaram pouca fidelidade entre as cepas testadas, capaz de formar bandas somente para $S$. Aureus e E. Coli, estas ainda de bandas de comprimentos consideravelmente distintos, 238 e 350 respectivamente, distinção semelhante entre bandas também fora encontrada no primer III, de resultado subóptimal. Estes resultados são respaldados na busca por um primer universal de maior eficiência realizado por Tekahashi27. Onde o primer VI (em seu estudo denominado 8F) fora rapidamente descartado para uso quando comparado aos outros primers testados.

\section{Agradecimentos}

Agradecemos a Universidade CEUMA por todo apoio e estrutura utilizados para execução desse trabalho, agradecemos também as agencias financiadoras FAPEMA e CAPES por fornecerem auxilio para a execução deste trabalho.

\section{Referências}

1. Challagundla $L$, Luo $X$, Tickler IA, Didelot X, Coleman DC, Shore AC, et al. Range Expansion and the Origin of USA300 North American Epidemic Methicillin-Resistant Staphylococcus aureus. mBio. 2018;9(1).

2. Hau SJ, Kellner S, Eberle KC, Waack U, Brockmeier SL, Haan JS, et al. Methicillin-Resistant Staphylococcus aureus Sequence Type (ST) 5 Isolates from Health Care and Agricultural Sources Adhere Equivalently to Human Keratinocytes. Applied and environmental microbiology. 2018;84(2).

3. Gillis HD, Demczuk WHB, Griffith A, Martin I, Warhuus M, Lang ALS, et al. PCR-based discrimination of emerging Streptococcus pneumoniae serotypes $22 \mathrm{~F}$ and $33 \mathrm{~F}$. Journal of microbiological methods. 2018;144:99-106.

4. Hare KM, Leach AJ, SmithVaughan HC, Chang AB, Grimwood K. Streptococcus pneumoniae and chronic endobronchial infections in childhood. Pediatric pulmonology. 2017;52(12):1532-45.

5. Santos-Juanes J, Fernandez-Vega I, Vivanco-Allende B, Fresno Forcelledo M. Perivascular basophilic rim as a histopathological clue of cutaneous Pseudomona aeruginosa sepsis. The American Journal of dermatopathology. 2015;37(5):4278.

6. Grundmann $\mathrm{H}$, Glasner $\mathrm{C}$, Albiger B, Aanensen DM, Tomlinson CT, Andrasevic AT, et al. Occurrence of carbapenemase-producing

Klebsiella pneumoniae and Escherichia coli in the European survey of carbapenemaseproducing Enterobacteriaceae (EUSCAPE): a prospective, multinational study. The Lancet 
Infectious

2017;17(2):153-63.

7. Hong KW, Asmah Hani AW, Nurul Aina Murni CA, Pusparani RR, Chong CK, Verasahib $\mathrm{K}$, et al. Comparative genomic and phylogenetic analysis of a toxigenic clinical isolate of Corynebacterium diphtheriae strain B-D-16-78 from Malaysia. Infection, genetics and evolution : journal of molecular epidemiology and evolutionary genetics in infectious diseases. 2017;54:263-70.

8. Stutz MD, Clark MP, Doerflinger M, Pellegrini M. Mycobacterium tuberculosis: Rewiring host cell signaling to promote infection. Journal of leukocyte biology. 2018;103(2):259-68.

9. Saputra S, Jordan D, Mitchell T, Wong HS, Abraham RJ, Kidsley A, et al. Antimicrobial resistance in clinical Escherichia coli isolated from companion animals in Australia. Veterinary microbiology. 2017;211:43-50.

10. Santos ALd, Santos DO, Freitas CCd, Ferreira BLA, Afonso IF, Rodrigues $\mathrm{CR}$, et al. Staphylococcus aureus: visitando uma cepa de importância hospitalar. Jornal Brasileiro de Patologia e Medicina Laboratorial. 2007;43:413-23.

11. Vieira AC, Gomes MC, Rolo Filho $M$, Eudes Filho J, Bello EJ, de Figueiredo RB. Streptococcus pneumoniae: a study of strains isolated from cerebrospinal fluid. Jornal de pediatria. 2007;83(1):718.

12. Sangal V, Tucker NP, Burkovski A, Hoskisson PA. The draft genome sequence of Corynebacterium diphtheriae bv. mitis NCTC 3529 reveals significant diversity between the primary diseasecausing biovars. Journal of bacteriology. 2012;194(12):3269.

13. Cerdeno-Tarraga AM, Efstratiou A, Dover LG, Holden MT, Pallen M,
Bentley SD, et al. The complete genome sequence and analysis of Corynebacterium diphtheriae NCTC13129. Nucleic acids research. 2003;31(22):6516-23.

14. Camus JC, Pryor MJ, Medigue C, Cole ST. Re-annotation of the genome sequence of Mycobacterium tuberculosis H37Rv. Microbiology. 2002;148(Pt 10):2967-73.

15. Glickman MS, Jacobs WR, Jr. Microbial pathogenesis of Mycobacterium tuberculosis: dawn of a discipline. Cell. 2001;104(4):477-85.

16. Reid G, Howard J, Gan BS. Can bacterial interference prevent infection? Trends in microbiology. 2001;9(9):424-8.

17. Eckburg PB, Bik EM, Bernstein CN, Purdom E, Dethlefsen L, Sargent $M$, et al. Diversity of the human intestinal microbial flora. Science. 2005;308(5728):1635-8.

18. Ebili HO, Hassall JC, Fadhil W, Ham-Karim H, Asiri A, Raposo TP, et al. "Squirrel" Primer-Based PCR Assay for Direct and Targeted Sanger Sequencing of Short Genomic Segments. Journal of biomolecular techniques : JBT. 2017;28(3):97-110.

19. Nathalang $O$, Intharanut $K$, Sasikarn W, Nathalang S, Kupatawintu P. A new polymerase chain reaction: sequence-specific primer method for the Augustine blood type. Blood transfusion = Trasfusione del sangue. 2016;14(6):577-9.

20. Spencer M, Barnes S, Parada J, Brown S, Perri L, Uettwiller-Geiger $D$, et al. A primer on on-demand polymerase chain reaction technology. American journal of infection control. 2015;43(10):1102-8.

21. Ritchie LE, Burke KF, GarciaMazcorro JF, Steiner JM, Suchodolski JS. Characterization of 
fecal microbiota in cats using universal 16S rRNA gene and group-specific primers for Lactobacillus and Bifidobacterium spp. Veterinary microbiology. 2010;144(1-2):140-6.

22. Saggu SK, Mishra PC. Characterization of thermostable alkaline proteases from Bacillus infantis SKS1 isolated from garden soil. PloS one. 2017;12(11):e0188724.

23. Mao DP, Zhou Q, Chen CY, Quan ZX. Coverage evaluation of universal bacterial primers using the metagenomic datasets. BMC microbiology. 2012;12:66.

24. Ito T, Suzaki K. Universal detection of phytoplasmas and Xylella spp. by TaqMan singleplex and multiplex real-time PCR with dual priming oligonucleotides. PloS one. 2017;12(9):e0185427.

25. Nadkarni MA, Martin FE, Jacques $N A$, Hunter N. Determination of bacterial load by real-time PCR using a broad-range (universal) probe and primers set. Microbiology. 2002;148(Pt 1):25766.

26. Hidalgo $M$, Prieto $I$, Abriouel $H$, Villarejo $A B$, Ramirez-Sanchez $M$, Cobo A, et al. Changes in Gut Microbiota Linked to a Reduction in Systolic Blood Pressure in Spontaneously Hypertensive Rats Fed an Extra Virgin Olive OilEnriched Diet. Plant foods for human nutrition. 2017.

27. Takahashi S, Tomita J, Nishioka K, Hisada T, Nishijima $M$. Development of a prokaryotic universal primer for simultaneous analysis of Bacteria and Archaea using next-generation sequencing. PloS one. 2014;9(8):e105592.

28. Bahl MI, Bergstrom A, Licht TR. Freezing fecal samples prior to DNA extraction affects the Firmicutes to Bacteroidetes ratio determined by downstream quantitative PCR analysis. FEMS microbiology 2012;329(2):193-7.

29. De Laveleye M, Huang TD, Bogaerts $\mathrm{P}$, Berhin C, Bauraing $\mathrm{C}$, Sacre $P$, et al. Increasing incidence of carbapenemase-producing Escherichia coli and Klebsiella pneumoniae in Belgian hospitals. European journal of clinical microbiology \& infectious diseases : official publication of the European Society of Clinical Microbiology. 2017;36(1):139-46.

30. Larsen N, Vogensen FK, van den Berg FW, Nielsen DS, Andreasen AS, Pedersen BK, et al. Gut microbiota in human adults with type 2 diabetes differs from nondiabetic adults. Plos one. 2010;5(2):e9085.

31. Bikandi, J., San Millán, R., Rementeria, A., and Garaizar, J. 2004. In silico analysis of complete bacterial genomes: PCR, AFLP$\mathrm{PCR}$, and endonuclease restriction. Bioinformatics 20:798-9. DOI: 10.1093/bioinformatics/btg491 